\title{
Noncommutative Gauge Theory on the $q$-deformed Euclidean Plane
}

\author{
Frank Meyer ${ }^{1,2}$ and Harold Steinacker ${ }^{2}$ \\ 1 Max-Planck-Institute for Physics (Werner-Heisenberg-Institut), Föhringer Ring \\ 6, D-80805 München, Germany meyerf@mppmu.mpg.de \\ 2 University of Munich, Theresienstr. 37, D-80333 München, Germany \\ hsteinac@theorie.physik. uni-muenchen.de
}

\section{Introduction}

The physical nature of space at very short distances is still not known. Already Heisenberg proposed in a letter to Peierls [1] that spacetime is quantized below some scale, suggesting that this could help to resolve the problem of infinities in quantum field theories. With this motivation, there has been a lot of work and progress in the formulation of quantum field theory on quantized or noncommutative spaces. Noncommutativity is implemented by replacing a differentiable space-time manifold by an algebra of noncommutative coordinates

$$
\left[x^{i}, x^{j}\right]=\theta^{i j}(x) \neq 0 .
$$

The simplest case is the so-called canonical quantum plane $\mathbb{R}_{\theta}^{n}$, where $\theta^{i j}$ is a constant tensor independent of $x$. This is the space which is usually considered in the literature [2]. However, most of the rotational symmetry is lost on $\mathbb{R}_{\theta}^{n}$. On the other hand, there exist quantum spaces which admit a generalized notion of symmetry, being covariant under a quantum group. Not much is known about field theory on this type of spaces.

One of the simplest spaces with quantum group symmetry is the Euclidean quantum plane $\mathbb{R}_{q}^{2}$. It is covariant with respect to the $q$-deformed twodimensional Euclidean group $E_{q}(2)$. We report here on our work [3], proposing a formulation of gauge theory based on the natural algebraic structures on this spaces and using a suitable star product.

\section{The $E_{q}(2)$-Symmetric Plane}

The $E_{q}(2)$-Symmetric Plane is generated by the complex coordinates $z, \bar{z}$ with the commutation relation

$$
z \bar{z}=q^{2} \bar{z} z
$$


We consider formal power series in these variables $z, \bar{z}$ as functions on this space,

$$
\mathbb{R}_{q}^{2}:=\mathbb{R}\langle\langle z, \bar{z}\rangle\rangle /\left(z \bar{z}-q^{2} \bar{z} z\right) .
$$

Notice that the simple commutation relation (2) are inconsistent with the usual formulas for differentiation and integration. We should therefore first discuss the appropriate differential calculus and invariant integration. Finally, to get physical predictions i.e. real numbers from the abstract algebra, we also need either a representation of the noncommutative algebra, or a realization of the algebra using a star product.

\subsection{Covariant Differential Calculus}

It is natural to require that there exist deformed spaces of $k$-forms $\Omega_{q}^{k}$ which are covariant with respect to $E_{q}(2)$, and that the exterior differential $d: \Omega_{q}^{k} \rightarrow$ $\Omega_{q}^{k+1}$ satisfies the usual Leibniz-rule as well as $d^{2}=0$. One can show that there exists a unique covariant differential calculus with these properties [4]:

$$
\begin{array}{ll}
z d z=q^{-2} d z z, & \bar{z} d z=q^{-2} d z \bar{z} \\
z d \bar{z}=q^{2} d \bar{z} z, & \bar{z} d \bar{z}=q^{2} d \bar{z} \bar{z}
\end{array}
$$

The following result is particularly useful for the construction of gauge field theories on $\mathbb{R}_{q}^{2}$ :

Lemma 1. Consider the one-forms

$$
\theta \equiv \theta^{z}:=z^{-1} \bar{z} d z \quad, \quad \bar{\theta} \equiv \theta^{\bar{z}}:=d \bar{z} z \bar{z}^{-1}
$$

and define

$$
\Theta:=\theta^{i} \lambda_{i}, \quad \text { where } \quad \lambda_{z}:=\frac{1}{1-q^{-2}} \bar{z}^{-1}, \lambda_{\bar{z}}:=-\frac{1}{1-q^{-2}} z^{-1} .
$$

Then for all functions $f \in \mathbb{R}_{q}^{2}$ and one-forms $\alpha$, the following holds:

$$
\begin{aligned}
{[\theta, f] } & =[\bar{\theta}, f]=0, \\
d f & =[\Theta, f]=\left[\lambda_{i}, f\right] \theta^{i} \\
d \alpha & =\{\Theta, \alpha\}
\end{aligned}
$$

denoting with $\{\cdot, \cdot\}$ the anti-commutator.

\subsection{Invariant Integral}

In order to define an invariant action, we need an integral on $\mathbb{R}_{q}^{2}$ which is invariant under quantum group transformations. This means that

$$
\int^{q} f(z, \bar{z}) \triangleleft X=\varepsilon(X) \int^{q} f(z, \bar{z})
$$


for all $f \in \mathbb{R}_{q}^{2}$ and $X \in U_{q}(e(2))$. Here $U_{q}(e(2))$ is the $q$-deformed universal enveloping Lie algebra of the two-dimensional Euclidean group, $\triangleleft$ denotes the right action of $U_{q}(e(2))$ on $\mathbb{R}_{q}^{2}$ and $\varepsilon(X)$ is the counit. Now any function in $\mathbb{R}_{q}^{2}$ can be decomposed as $f(z, \bar{z})=\sum_{m \in \mathbb{Z}} z^{m} f_{m}(z \bar{z})$. It can be shown that (10) is satisfied for the following discrete quantum traces [5]

$$
\int^{q,\left(r_{0}\right)} f(z, \bar{z}):=r_{0}^{2}\left(q^{2}-1\right) \sum_{k=-\infty}^{\infty} q^{2 k} f_{0}\left(q^{2 k} r_{0}^{2}\right),
$$

where $r_{0} \in \mathbb{R}$ labels the irreducible representations of $\mathbb{R}_{q}^{2}$. The most general invariant integral is given by superpositions of these integrals,

$$
\int^{q} f(z, \bar{z})=\int_{1}^{q} d r_{0} \mu\left(r_{0}\right) \int^{q,\left(r_{0}\right)} f_{0}(z \bar{z})
$$

with arbitrary "weight" function $\mu(r)>0$. It is quite remarkable and useful that for the special choice $\mu\left(r_{0}\right)=\frac{1}{r_{0}\left(q^{2}-1\right)}$, one recovers the usual Riemannian integral, which is therefore also invariant under $U_{q}(e(2))[3]$.

\section{Star Product Approach}

\subsection{The Star Product}

The noncommutative algebra $\mathbb{R}_{q}^{2}$ can be realized on the algebra of commutative functions on $\mathbb{R}^{2}$ using a new, noncommutative product, called star product. Let us denote the commutative variables on $\mathbb{R}^{2}$ by greek letters $\zeta, \bar{\zeta}$ to distinguish them from the generators $z, \bar{z}$, and let $q=: e^{h}$. Then a hermitian star product for $\mathbb{R}_{q}^{2}$ is given by

$$
f \star g:=\mu \circ e^{h\left(\zeta \partial_{\zeta} \otimes \bar{\zeta} \partial_{\bar{\zeta}}-\bar{\zeta} \partial_{\bar{\zeta}} \otimes \zeta \partial_{\zeta}\right)}(f \otimes g)=f g+h \bar{\zeta}\left(\partial_{\zeta} f \partial_{\bar{\zeta}} g-\partial_{\bar{\zeta}} f \partial_{\zeta} g\right)+\mathcal{O}\left(h^{2}\right) .
$$

\subsection{Noncommutative Gauge Transformations}

The formalism of covariant coordinates was established in [6] for an arbitrary Poisson structure. This leads to problems in the semi-classical limit ${ }^{3}$. Therefore we propose the following approach, taking advantage of the frame $\theta, \bar{\theta}$ which commutes with all functions and the generator $\Theta$ of the exterior differential. We define infinitesimal gauge transformations of a matter field as

\footnotetext{
${ }^{3}$ To obtain in the classical limit the classical gauge field $a_{i}$ we have to invert $\theta^{i j}$. This is only well-defined if $\theta$ is invertible, and even then it spoils the covariant transformation property whenever $\theta$ is not constant. To maintain covariance one has to "invert $\theta$ covariantly" as done in [7], leading to complicated expressions.
} 


$$
\delta \psi=i \Lambda \star \psi, \quad \delta \zeta^{i}=0 .
$$

Let us introduce the "covariant derivative" (or covariant one-form) as

$$
D:=\Theta-i A \text {. }
$$

Then requiring that $D \psi$ transforms covariantly, i.e.

$$
\delta D \star \psi \stackrel{!}{=} i \Lambda \star D \star \psi
$$

leads to the following gauge transformation property for the gauge field $A$ :

$$
\delta A=[\Theta \stackrel{\star}{,}, \Lambda]+i[\Lambda \stackrel{\star}{,} A]=d \Lambda+i[\Lambda \stackrel{\star}{,} A] .
$$

We define the field strength as the two-form

$$
F:=D \wedge_{q} D
$$

where $\wedge_{q}$ the star-wedge [3]. Then

$$
\delta F=i[\Lambda \stackrel{\star}{, F}] .
$$

As a two-form, the field strength can be written as $F=f \theta \wedge_{q} \bar{\theta}$. Since the frame $\theta, \bar{\theta}$ commutes with functions, $f$ transforms covariantly as well:

$$
\delta f=i[\Lambda \stackrel{\star}{*} f] .
$$

We note that all transformations have the correct classical limit as $q \rightarrow 1$.

\subsection{Seiberg-Witten Map}

The Seiberg-Witten map [8] allows to express the noncommutative gauge fields in terms of the commutative ones. Hence the noncommutative theory can be interpreted as a deformation of the commutative theory. Its physical predictions can be explicitly obtained by expanding in the deformation parameter $h$, and the commutative theory is reproduced in the limit $h \rightarrow 0$. The Seiberg-Witten map is based on the following requirement:

- The consistency condition:

$$
\begin{aligned}
\left(\delta_{\alpha} \delta_{\beta}-\delta_{\beta} \delta_{\alpha}\right) \Psi & =\delta_{-i[\alpha, \beta]} \Psi \\
\Leftrightarrow i \delta_{\alpha} \Lambda_{\beta}-i \delta_{\beta} \Lambda_{\alpha}+\left[\Lambda_{\alpha}^{\star}, \Lambda_{\beta}\right] & =i \Lambda_{-i[\alpha, \beta]},
\end{aligned}
$$

- Noncommutative gauge transformations are related to commutative ones:

$$
\begin{aligned}
A_{i}\left[a_{i}\right]+\delta_{\Lambda} A_{i}\left[a_{i}\right] & =A_{i}\left[a_{i}+\delta_{\alpha} a_{i}\right] \\
\Psi\left[\psi, a_{i}\right]+\delta_{\Lambda} \Psi\left[\psi, a_{i}\right] & =\Psi\left[\psi+\delta_{\alpha} \psi, a_{i}+\delta_{\alpha} a_{i}\right] .
\end{aligned}
$$

Solving these conditions in our case, we obtain the following expression for the field strength expanded in powers of $h$ :

$$
\begin{aligned}
f= & F_{12}^{0}+h\left\{F_{12}^{0}+\theta^{12}\left(F_{12}^{0} F_{12}^{0}-a_{\zeta} \partial_{\bar{\zeta}} F_{12}^{0}+a_{\bar{\zeta}} \partial_{\zeta} F_{12}^{0}\right)+\partial_{\zeta} \theta^{12}\left(a_{\zeta} \partial_{\bar{\zeta}} a_{\bar{\zeta}} \quad(23)\right.\right. \\
& \left.\left.+a_{\bar{\zeta}} \partial_{\bar{\zeta}} a_{\zeta}+2 a_{\bar{\zeta}} \partial_{\zeta} a_{\bar{\zeta}}\right)+\partial_{\bar{\zeta}} \theta^{12}\left(a_{\zeta} \partial_{\zeta} a_{\bar{\zeta}}+a_{\bar{\zeta}} \partial_{\zeta} a_{\zeta}+2 a_{\zeta} \partial_{\bar{\zeta}} a_{\zeta}\right)\right\}+\mathcal{O}\left(h^{2}\right),
\end{aligned}
$$

where $a_{\zeta_{i}}$ is the classical gauge field and $F_{i j}^{0}=\partial_{\zeta_{i}} a_{j}-\partial_{\zeta_{j}} a_{i}$ is the classical field strength. 


\subsection{The Action}

To define a gauge-invariant action, we need an integral which is cyclic with respect to the star product, since the field strength transforms in the adjoint. The invariant integrals (11) do not have this property, which can be restored by introducing a measure function $\mu$. It can be shown that for the measure function $\mu:=\frac{1}{\zeta \zeta}$, we can even drop the star under the integral:

$$
\int d \zeta d \bar{\zeta} \mu f \star g=\int d \zeta d \bar{\zeta} \mu f g=\int d \zeta d \bar{\zeta} \mu g \star f
$$

Putting all this together, we can now define a gauge invariant action:

$$
S:=\frac{1}{2} \int d \zeta d \bar{\zeta} \mu f \star f .
$$

Gauge invariance is guaranteed because of (20). Moreover, the classical action for abelian gauge field theory is reproduced in the classical limit $h \rightarrow 0$ because of (23).

Another possibility is to replace the measure function $\mu$ by a scalar "Higgs" field $\varphi$, which transforms such that it restores the gauge invariance of the action. One can in fact find a suitable potential for $\phi$ which admits a solution $\langle\phi\rangle=\mu=\frac{1}{\zeta \zeta}$, leading to a $E_{q}(2)$-invariant action through spontaneous symmetry breaking.

\section{References}

1. W. Pauli: Scientific Correspondence, Vol. II (Springer 1985) p 15.

2. M. R. Douglas and N. A. Nekrasov: Rev. Mod. Phys. 73, 977 (2001).

3. F. Meyer and H. Steinacker: Int.J.Mod.Phys. A19, 3349-3376 (2004).

4. M.Chaichian and A. P. Demichev: Phys. Lett. B320, 273 (1994).

5. H. T. Koelink: Duke Math. J. 76, 483 (1994).

6. J. Madore, S. Schraml, P. Schupp and J. Wess: Eur. Phys. J. C16, 161 (2000).

7. F. Meyer: Models of Gauge Field Theory on Noncommutative Spaces, DiplomaThesis, Univ. of Munich, Chair Prof. J. Wess (2003), arXiv: hep-th/0308186.

8. N. Seiberg and E. Witten: JHEP 09, 032 (1999). 